University of Nebraska - Lincoln

DigitalCommons@University of Nebraska - Lincoln

Faculty Publications, Department of Psychology

Psychology, Department of

6-2009

\title{
Going Beyond the Specifics: Generalization of Single Actions, But Not Temporal Order, at 9 Months
}

\author{
Angela F. Lukowski \\ University of California-Irvine, angela.lukowski@uci.edu \\ Sandra A. Wiebe \\ University of Nebraska-Lincoln, sandra.wiebe@ualberta.ca \\ Patricia J. Bauer \\ Emory University, patricia.bauer@emory.edu
}

Follow this and additional works at: https://digitalcommons.unl.edu/psychfacpub

Part of the Psychiatry and Psychology Commons

Lukowski, Angela F.; Wiebe, Sandra A.; and Bauer, Patricia J., "Going Beyond the Specifics: Generalization of Single Actions, But Not Temporal Order, at 9 Months" (2009). Faculty Publications, Department of Psychology. 381.

https://digitalcommons.unl.edu/psychfacpub/381

This Article is brought to you for free and open access by the Psychology, Department of at DigitalCommons@University of Nebraska - Lincoln. It has been accepted for inclusion in Faculty Publications, Department of Psychology by an authorized administrator of DigitalCommons@University of Nebraska - Lincoln. 
Published in Infant Behavior and Development 32:3 (June 2009), pp. 331-335; doi: 10.1016/j.infbeh.2009.02.004

Copyright (c) 2009 Elsevier Inc. Used by permission. http://www.elsevier.com/locate/inbede

Submitted May 12, 2008; revised January 12, 2009; accepted February 25, 2009; published online March 272009.

\title{
Going beyond the specifics: Generalization of single actions, but not temporal order, at 9 months
}

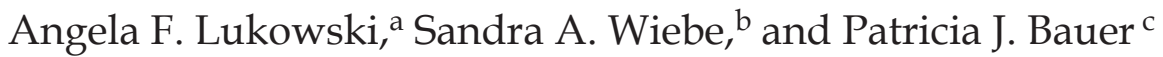 \\ ${ }^{\text {a }}$ Department of Psychology and Social Behavior, University of California-Irvine, \\ 3340 Social Ecology Building II, Irvine, CA 92697, USA \\ ${ }^{b}$ Department of Psychology, University of Nebraska-Lincoln, Lincoln, NE, USA \\ ${ }^{c}$ Department of Psychology, Emory University, Atlanta, GA, USA \\ Corresponding author - A. F. Lukowski,
}

tel 949 824-7191, fax 949 824-3002, email angela.lukowski@uci.edu

\begin{abstract}
We examined generalization in 9-month-old infants after a 24-hour delay using deferred imitation. Infants flexibly applied their knowledge of sequence actions across changes in props even though they had no opportunity for immediate imitation.
\end{abstract}

Keywords: memory, generalization, imitation, infancy

The functional significance of memory lies in the balance between remembering the specific features of particular experiences and flexibly applying acquired knowledge across different cues and contexts. By the time children are 21 months of age, generalization across cues is robust over delays of $24 \mathrm{~h}$, even when the stimuli presented at encoding and test are largely dissimilar (Hayne, MacDonald, \& Barr, 1997). Generalization also is apparent at the end of the first year of life when infants are allowed the opportunity for immediate recall with the original stimuli (Learmonth, Lamberth, \& Rovee-Collier, 2004; McDonough \& Mandler, 1998) or when the stimuli used at demonstration are present when generalization is tested (Baldwin, Markman, \& Melartin, 1993). The current study was conducted to determine whether 9-month-old infants generalize learning across distinct materials after a 24-h delay without the benefit of immediate recall or ongoing perceptual support from the original materials.

In the second half of the first year of life and beyond, generalization across cues has largely been studied using imitation paradigms. In this technique, participants witness an experimenter demonstrate a novel sequence of actions. After a delay, participants are given perceptually distinct yet functionally identical analogue versions of the stimuli and are encouraged to interact with the props. The question is whether they use the analogue versions to produce the demonstrated sequence. Use of this method has revealed the developmental progression of generalization over the first and second years of life. Specifically, cross-sectional research has indicated that 12-month-old do not generalize learned information to analogue stimuli differing only in color after a 24-h delay. By 18 months of age, generalization is apparent after $24 \mathrm{~h}$ when analogue items differ in color, but not when they differ in both color and shape. Generalization across stimuli that vary on both dimensions first becomes apparent when children are approximately 21 months old (Hayne et al., 1997). These data suggest that generalization across cues after a delay is largely absent in imitation paradigms until the middle of the second year, even when analogue test materials closely resemble those seen at encoding. 
However, generalization across dissimilar materials has also been demonstrated in the first year of life when certain mnemonic supports are provided. In an imitation-based inductive reasoning task, 9-month-old infants appropriately generalized domain-specific properties (e.g., drinking or going for a ride) to novel exemplars of the same category when the generalization test followed an opportunity for immediate imitation using the original objects (McDonough \& Mandler, 1998). Infants of the same age also immediately generalized a novel action that they discovered through exploratory play when the original item was left on the table for active comparison with the analogue (Baldwin et al., 1993). Unfortunately, these two studies did not include conditions to test whether infants would also generalize in the absence of immediate imitation or ongoing perceptual support from the original item. This distinction is important, in that 9-month-old infants show evidence of 24-h delayed generalization in an imitation task using highly distinct puppets when they are allowed the opportunity for immediate imitation, but not when it is prohibited (Learmonth et al., 2004). The present study was conducted to determine whether 9-month-old infants generalize across stimuli after a 24-h delay under conditions of purely deferred imitation. The ability to defer generalization closely parallels life outside the laboratory: infants who are able to abstract information from familiar cues may be able to generalize that knowledge to novel ones, thereby allowing them greater opportunity to learn about the world in which they live.

Fifteen 9.5-month-old infants (mean age $=9$ months, 12 days; range from 9 months, 7 days to 9 months, 24 days; 8 girls) were recruited from a participant database maintained by a large Midwestern university. The pool includes infants whose parents were contacted by mail shortly after their infants' births and subsequently returned informational cards stating their willingness to be contacted about potential participation in research. All of the participants in the present study were born at term ( $40 \pm 2$ weeks gestational age) and were experiencing an apparently normal course of development.

Each infant participated in two exposure sessions and one delayed recall session. Infants were allowed two exposures to the two-step sequences to allow them the best opportunity to generalize, as multiple exposures facilitate longterm memory at the end of the first year of life (Bauer, Wiebe, Waters, \& Bangston, 2001). Each sequence was constrained by enabling relations, such that the actions had to be completed in order for a desirable end-state to be achieved (though as in Bauer, Wiebe, Carver, Waters, \& Nelson, 2003; Carver \& Bauer, 1999; Carver \& Bauer, 2001, for example, it was physically possible to produce the actions in any order). Sequences constrained by enabling relations allow infants the best opportunity for recall. Indeed, children younger than 20 months of age perform at chance on memory for the temporal order of arbitrarily ordered sequences (Wenner \& Bauer, 1999). At the first exposure session, infants interacted with 6 out of a pool of 14 novel two-step sequences for a timed baseline period lasting between 1.5 and 2 min. Immediately thereafter, an experimenter modeled each sequence of actions twice in succession, with narration. At the second exposure session (mean delay $=1$ day, range 1-2 days), the experimenter modeled the same six sequences twice more with narration in a different counterbalanced order. Immediate imitation was not permitted at either session.

At the delayed recall session (mean delay = 1 day; range 1-2 days), infants were presented with the materials necessary to complete six sequences. Two sequences were tested in each of three conditions (see Figure 1 for examples). In the familiar condition, the materials were the same props that had been used in the experimenter's demonstration. In the analogue condition, the props were perceptually distinct yet functionally identical to those used by the experimenter. In the novel condition, the props were perceptually and functionally dissimilar to any that had been used previously. The novel condition was included as a control to test whether increased performance from baseline to delayed recall on familiar and analogue sequences could be attributed to learning from the experimenter's demonstration or resulted from non-specific factors such as familiarity with deferred imitation testing. The test procedure for all conditions was identical: the experimenter gave the infant the props and provided the name of the sequence that could be performed. The sequence label served as a verbal reminder for sequences tested in the familiar and analogue conditions and as a non-specific prompt for sequences in the novel condition.

The first and third sessions were videotaped for analysis. An experienced behavioral coder who was unaware of the hypotheses of the study coded the behavior of each infant, including both the occurrence of target actions and their order. Only the first occurrence of each behavior was coded so as to reduce the likelihood of credit for behaviors produced by chance or trial and error, thereby providing the most conservative measure of ordered recall. A second coder independently recoded the tapes for four infants (27\% of the sample). Mean percent agreement was $88 \%$ (range $81-94 \%)$ for the occurrence of target actions and their order.

We conducted separate one-way within-subjects analyses of variance (ANOVAs) by phase (baseline and delayed recall) for each of the three conditions (familiar, analogue and novel) to determine whether infants evidenced increased performance on target actions at delayed recall relative to baseline. This comparison is necessary to determine whether infants' behavior was based on memory (possible at delayed recall only) or whether it could be attributed to trial-and-error problem solving (possible at baseline). As shown in Figure 2, panel A, infants performed a greater number of target actions at delayed recall relative to baseline on familiar, $F(1,14)=8.71, p<.01$, and analogue sequences only, $F(1,14)=16.00, p<.001$; phase differences were not apparent on novel sequences $(p=.23)$. Thus, there was evidence that infants generalized single actions after a 24-h delay.

Because only two infants produced any ordered pairs in any condition at baseline, our analysis of memory for and generalization of temporal order information could not include a baseline comparison. As such, we adopted a different statistical approach. We conducted a one-way within-subjects comparison of performance at delayed recall by condition (familiar, analogue and novel) on the production of ordered pairs at delayed recall only, reasoning that in- 

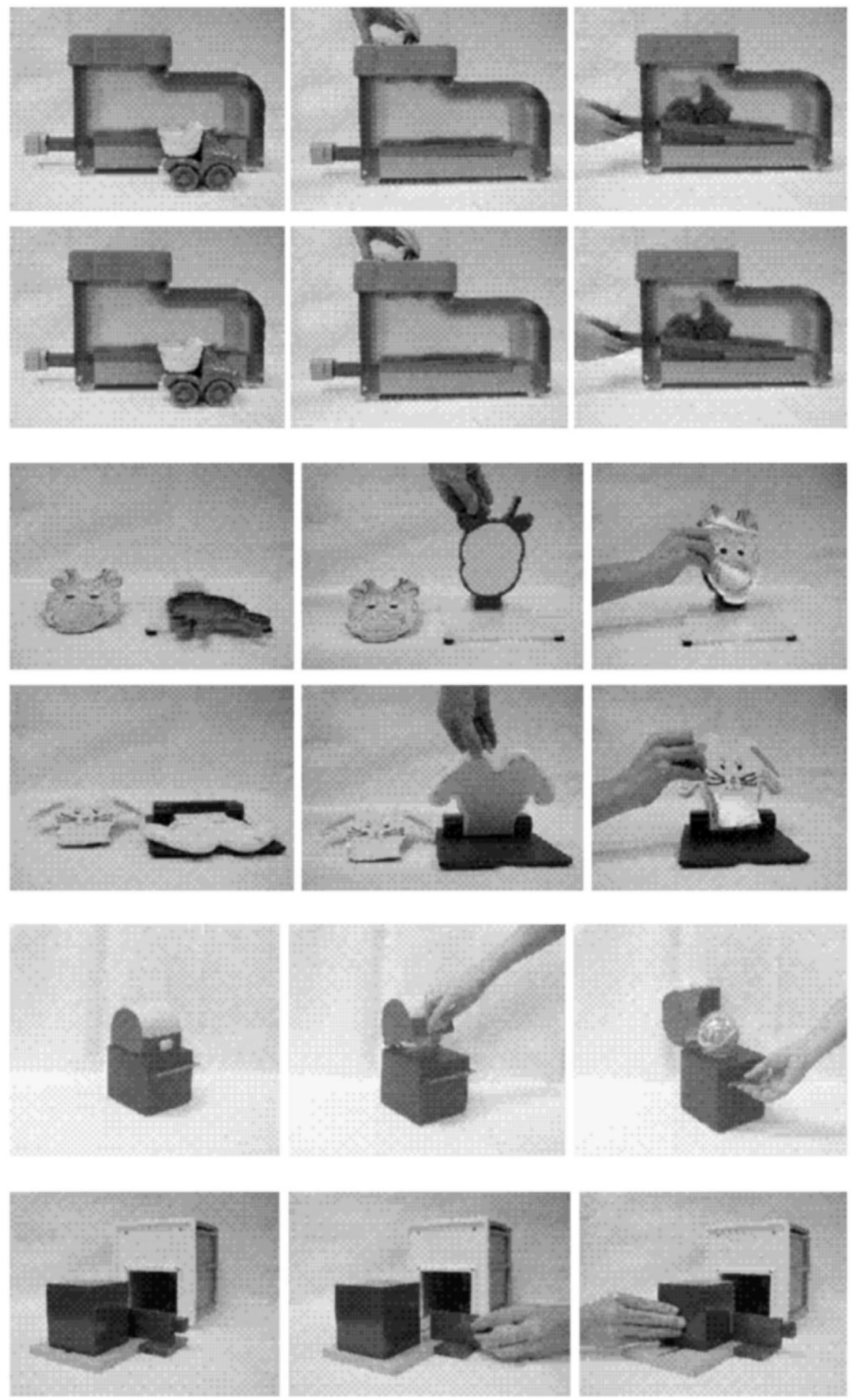

Figure 1. Examples of familiar, analogue, and novel sequences are shown in panels A, B, and C, respectively. The first sequence in each panel shows a sequence presented at the first two familiarization sessions; the second sequence in each panel depicts the materials available at the third delayed recall session. From the left, the photographs in each row show the first step, the second step, and all of the props used to complete each sequence.

fants should show increased levels of performance for sequences that were better retained in memory. As shown in Figure 2, panel B, a significant main effect of condition was found: $F(2,28)=3.50, p<.04$. Follow-up Tukey tests indicated that infants performed significantly more pairs of actions at delayed recall on familiar relative to novel sequences; performance on analogue sequences was intermediate between the two. ${ }^{1}$

${ }^{1}$ Correlations conducted between continuous delay in hours (from the first to the second and from the second to the third sessions) with the production of target actions and pairs of actions at delayed recall indicated that the variable delay between sessions did not influence performance on sequences tested in any condition. 

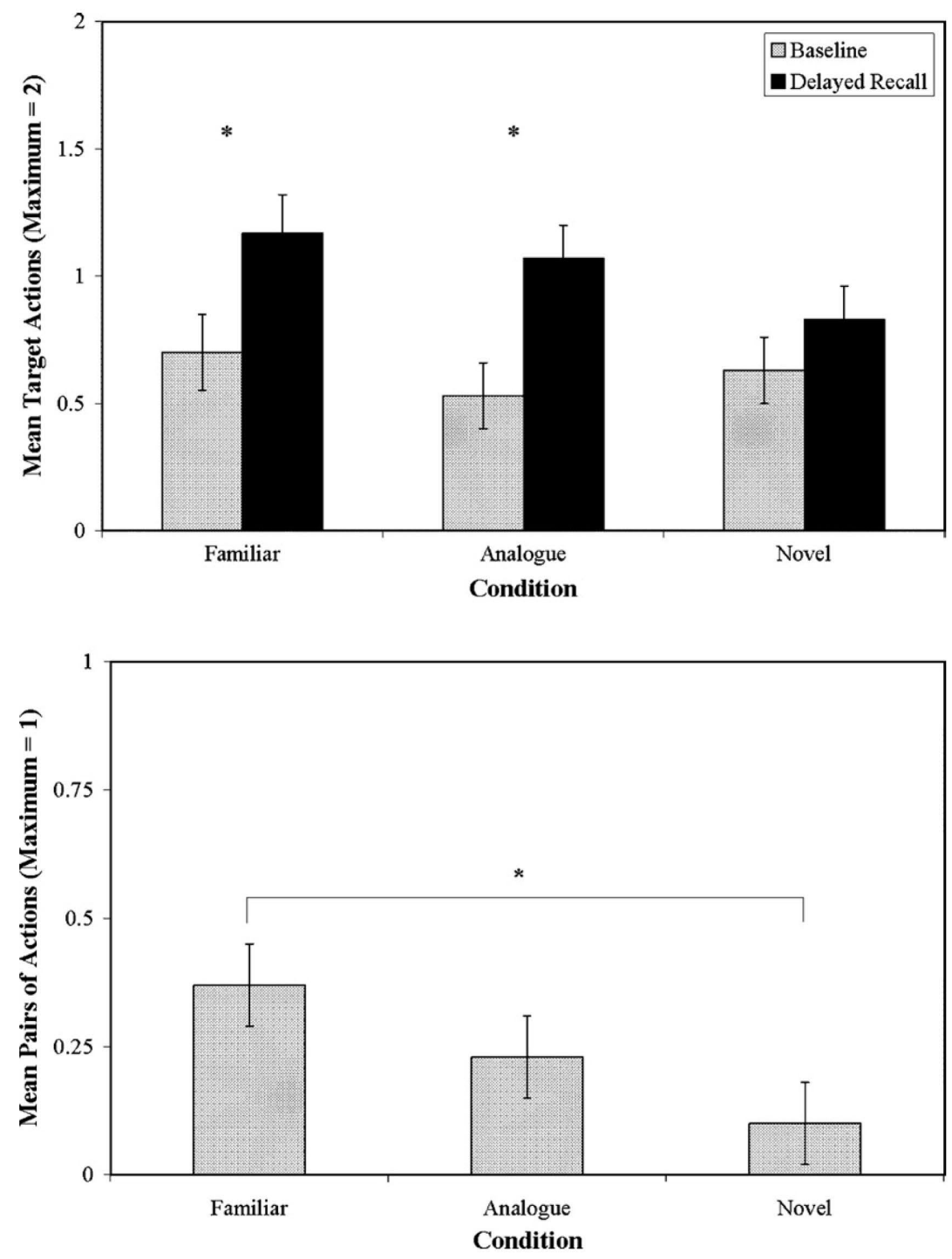

Figure 2. The number of target actions produced by condition and phase (panel A) and the number of pairs of actions produced in the correct temporal order by condition at delayed recall (panel B).

The obtained pattern of results suggests that 9-month-olds generalized individual target actions, but not temporal order information, after a 24-h delay. That is, infants performed a greater number of target actions on familiar and analogue, but not on novel sequences, at delayed recall relative to baseline. The completion of pairs of actions for familiar sequences also exceeded that found for novel ones tested at the same session, thereby indicating that infants remembered the specific temporal order information for the sequences on which they were tested. However, performance on analogue sequences was intermediate between, and not statistically different from, performance on familiar or novel sequences, thereby indicating that infants failed to generalize temporal order information to perceptually distinct, functionally identical materials.

The present finding of generalization of single target actions after a 24-h delay without the benefit of immediate imitation or ongoing perceptual support from the original materials contributes to our understanding of memory development near the end of the first year of life. There is mounting evidence that long-term memory is relatively fragile at 9 months of age: even when given multiple exposures to two-step sequences, 9-month-olds as a group fail to recall temporal order after a 1-month delay (Bauer et al., 2001, 2003; Carver \& Bauer, 1999). In contrast, this effect is robust and statistically reliable when infants are 10.5 months old (Carver \& Bauer, 2001). Nevertheless, multiple exposures appear to support the establishment of more robust memory representations: 9-month-olds given three exposures to two-step sequences recalled single target actions and pairs of actions after a 1-month delay, whereas infants given 1 or 2 exposures only recalled individual actions (Bauer et al., 2001). The extent to which repeated exposure impacted the 24-h delayed generalization capabilities of 9-month-olds in the present study remains to be determined through future research, but could plausibly explain the obtained pattern of results. 
In the present study, after two exposures, 9-month-old infants generalized their learning of individual target actions but not temporal order information to perceptually distinct, functionally identical event sequences. It is possible that generalization in 9-month-olds is critically dependent on the strength of the established memory representation: it has been suggested that memory representations that vary in strength support different levels of behavioral competence (e.g., Munakata, 2001). Infants' representations of sequences they have observed on two occasions appear to support generalization of actions, but not generalized ordered recall, which has previously been shown to place higher demands on memory (Bauer, Wenner, Dropik, \& Wewerka, 2000). Within this framework, experimental manipulations that increase infants' ability to encode, consolidate, and access stored information would result in improved generalization of both target actions and temporal order information. Consistent with this suggestion, allowing infants of this age the opportunity for immediate imitation previously has been shown to improve both 24-h delayed generalization (Learmonth et al., 2004) and 1-month delayed recall, with benefits apparent for temporal order information (Lukowski et al., 2005).

The finding that 9-month-old infants can generalize information after a 24-h delay has theoretical implications for the study of cognitive development. It indicates that the memory representations of 9-month-olds are not necessarily constrained to the specific features of the stimuli observed during encoding but also can be flexibly applied to novel stimuli that differ in both color and form. However, infants' ability to generalize may depend on the strength of their memory representations. There also are functional implications for infants' conceptual development: as infants begin to self-locomote and independently explore the environment, their ability to generalize should permit them to extrapolate learned information to novel cues that resemble those encountered previously, thereby broadening and enriching their knowledge of the world around them and permitting them to react appropriately to novel situations (see Herbert, Gross, \& Hayne, 2007).

\section{Acknowledgments}

This research was completed while all of the authors were at the Institute of Child Development, University of Minnesota. This research was supported by a grant from the NICHD (HD-28425) to Patricia J. Bauer; support for Angela F. Lukowski was provided by a Ruth L. Kirschstein National Research Service Award (NRSA) from NIMH (1 F31 MH072110-02); and support for Sandra A. Wiebe was provided by a National Science and Engineering Research Council of Canada (NSERC) fellowship (PGSB-242658-2001). We are grateful to the participants and their families who gave so generously of their time to participate in this research, and to the members of the Cognition in the Transition Laboratory for assistance with data collection and coding. Portions of these data were presented at the 71st biennial meeting of the Society for Research in Child Development, Atlanta, GA, 2005. A more complete report of these data will be provided upon request.

\section{References}

Baldwin et al., 1993 D. A. Baldwin, E. M. Markman, and R. L. Melartin, Infants' ability to draw inferences about nonobvious object properties: Evidence from exploratory play, Child Development 64 (1993), pp. 711-728.

Bauer et al., $2000>$ P. J. Bauer, J. A. Wenner, P. L. Dropik, and S. S. Wewerka, Parameters of remembering and forgetting in the transition from infancy to early childhood, Monographs of the Society for Research in Child Development $65(2000)(4$, serial no. 263).

Bauer et al., 2003 P. J. Bauer, S. A. Wiebe, L. J. Carver, J. M. Waters, and C. A. Nelson, Developments in long-term explicit memory late in the first year of life: Behavioral and electrophysiological indices, Psychological Science 14 (2003), pp. 629-635.

Bauer et al., 2001 P. J. Bauer, S. A. Wiebe, J. M. Waters, and S. K. Bangston, Reexposure breeds recall: Effects of experience on 9month-olds' ordered recall, Journal of Experimental Child Psychology 80 (2001), pp. 174-200.

Carver and Bauer, 1999 L. J. Carver and P. J. Bauer, When the event is more than the sum of its parts: 9-month-olds' long-term ordered recall, Memory 7 (1999), pp. 147-174.

Carver and Bauer, 2001 L. J. Carver and P. J. Bauer, The dawning of a past: The emergence of long-term explicit memory in infancy, Journal of Experimental Child Psychology: General 130 (2001), pp. 726-745.

Hayne et al., $1997-$ H. Hayne, S. MacDonald, and R. Barr, Developmental changes in the specificity of memory over the second year of life, Infant Behavior and Development 19 (1997), pp. 233-245.

Herbert et al., 2007 J. Herbert, J. Gross, and H. Hayne, Crawling is associated with more flexible memory retrieval by 9-month-old infants, Developmental Science 10 (2007), pp. 183-189.

Learmonth et al., 2004 A. E. Learmonth, R. Lamberth, and C. Rovee-Collier, Generalization of deferred imitation during the first year of life, Journal of Experimental Child Psychology 88 (2004), pp. 297-318.

Lukowski et al., 2005 A. F. Lukowski, S. A. Wiebe, J. C. Haight, J. M. Waters, C. A. Nelson, and P. J. Bauer, Forming a stable memory representation in the first year of life: Why imitation is more than child's play, Developmental Science 8 (2005), pp. 279-298.

McDonough and Mandler, 1998 L. McDonough and J. M. Mandler, Inductive generalization in 9- and 11-month-olds, Developmental Science 1 (1998), pp. 227-232.

Munakata, 2001 Y. Munakata, Graded representations in behavioral dissociations, Trends in Cognitive Sciences 5 (2001), pp. 309-315.

Wenner and Bauer, 1999 J. A. Wenner and P. J. Bauer, Bringing order to the arbitrary: One- to two-year-olds' recall of event sequences, Infant Behavior and Development 22 (1999), pp. 585-590. 